\title{
Unraveling the mechanisms involved in human norovirus pathogenesis: An update in norovirus pathogenesis
}

\author{
Magda E. Vera-Garcia ${ }^{1,2}$, Natalia Martinez-Acuña ${ }^{1,2}$, Sonia A. Lozano-Sepulveda ${ }^{1,2}$, \\ Daniel Arellanos-Soto ${ }^{1,2}$, Kame A. Galan-Huerta ${ }^{1,2}$, José A. Merino-Mascorro ${ }^{3}$, Javier Ramos-Jimenez ${ }^{1,2}$, \\ Norma Heredia ${ }^{3}$, and Ana $M^{a}$ Rivas-Estilla ${ }^{1,2 *}$ \\ ${ }^{1}$ Department of Biochemistry and Molecular Medicine, School of Medicine; ${ }^{2}$ Center of Research and Innovation on Medical Virology, School of \\ Medicine; ${ }^{3}$ Department of Microbiology and Immunology, School of Biology, Universidad Autónoma de Nuevo León, Monterrey, Nuevo Leon, Mexico
}

\begin{abstract}
RNA viruses causing human diseases impinge public health and the worldwide economy, as are the case of the current SARS-CoV-2 pandemic. These infectious pathogens are well known to control cellular machinery to complete their replication cycle, specifically taking advantage of the endoplasmic reticulum membrane to build replication complexes (RCs). Norovirus (NV) is a major cause of human gastroenteritis worldwide; this emerging RNA virus is a small positive sense non-enveloped virus belonging to the family Caliciviridae. Recent advances in the development of NV study models have led to an increased understanding of NV replication, pathogenesis, cell tropism, and host immunity response; nonetheless, it remains a challenging pathogen to study due to the lack of a robust model for human NV propagation. Therefore, its pathogenesis is still under characterization. Here, we present an approach to the molecular mechanisms disturbing cellular homeostasis induced by viral protein interactions to specific usage of unfolded protein response and proteasome, cellular processes induced by endoplasmic reticulum stress.
\end{abstract}

Key words: Norovirus. Endoplasmic reticulum. Unfolded protein response. Proteasome. RNA virus. Acute gastroenteritis.

\section{Introduction}

Noroviruses (NVs) are the most important cause of acute gastroenteritis (AGE) worldwide, estimating 677 million cases annually and 200,000 deaths in the world ${ }^{1}$. NV infections are currently a public health issue due to its high prevalence and mortality rates reported for children, elders, and immune depressed patients ${ }^{2}$; mainly in lower-middle- and low-income countries. This viral pathogen predominantly replicates in enterocytes ${ }^{3}$, causing AGE characterized by watery diarrhea and/or vomiting in children and adults with a sudden onset accompanied by signs such as abdominal cramps, nausea, and fever ${ }^{4,5}$.
Despite its clinical relevance, the study of human noroviruses (HuNVs) has been limited by a lack of study models ${ }^{3,6-8}$; therefore, NV proteins are still under characterization and their molecular pathogenic mechanisms are yet to be defined.

This review discusses current advances in the endoplasmic reticulum (ER) stress molecular mechanisms involved in NV infection.

\section{NV genome and replication}

NV is small non-enveloped positive-single-strand RNA virus members of the Caliciviridae family ${ }^{9}$. NV is

\section{Correspondence:}

*Ana Ma Rivas-Estilla

E-mail: amrivas1@yahoo.ca
Available online: 01-12-2021

Date of acceptance: 01-10-2021 DOI: 10.24875/RMU.21000032
Medicina Universitaria. 2021;23(4):142-147 www.medicinauniversitaria.org

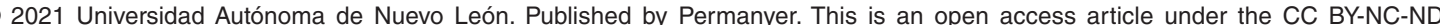
license (http://creativecommons.org/licenses/by-nc-nd/4.0/). 
subdivided into 10 genogroups (GI-GX) from which genogroups G.I., GII, and GIV can infect humans ${ }^{10}$; GII is associated with $80-90 \%$ of NV infections, with subtype Gll.4 currently being found in $70 \%$ of NV cases and outbreaks ${ }^{11}$. Its viral genome ranges from 7.5 to $8.5 \mathrm{~kb}$ in length, with a short five nucleotide $5^{\prime}$ UTR covalently linked to the viral protein called VPg, a short 3'UTR with a poly-A tail, and a long coding sequence for all viral proteins. The coding sequence includes ORF1, which is separated from ORF2 and ORF3. An additional ORF4 comprised within ORF2 was found only in murine NV (MNV), encoding a virulence factor (Fig. 1) ${ }^{9}$.

In addition, the NV replication cycle comprises a sequence of steps initiated by virus binding to the cell surface using various carbohydrate attachment factors, such as cell-associated host glycans such as sialic acid $^{12}$, histo-blood group antigens ${ }^{13}$, and soluble cofactors $^{14}$ for the entry of MNV and HuNV.

The second stage of viral entry is receptor engagement $^{15-18}$, where the human receptor remains unidentified. Following receptor engagement, the virus enters the cell through endocytosis, where receptor binding triggers the minor capsid protein VP2 to form a membrane portal that enables viral genome release in the cytoplasm $^{19}$. Subsequently, the RNA genome is translated at the boundaries of the rough ER (RER $)^{10}$. After the complete set of viral proteins is synthesized, the RC is established, allowing genomic and subgenomic viral replication. Once subgenomic RNA is produced, capsid proteins are translated and eventually assembled into viral particles.

\section{Viral infections and ER stress}

The ER is a fundamental organelle for cell function and survival. Spanning the nuclear envelope to the cell membrane, this organelle is organized in subdomains of different shapes, such as tubules and cisternae, giving rise to the smooth ER (SER) and RER, with the last one being complemented by ribosomes transiently attached to the external side. Under normal conditions, ER functions involve fundamental regulating processes for cell homeostasis. While the RER is mainly responsible for the correct folding and posttranslational modification of proteins and their exportation, lipids to be delivered to other intracellular organelles are synthesized in the SER ${ }^{20}$. When the ER protein-folding capacity is disturbed, cells suffer misfolded protein accumulation inside the ER lumen, causing ER stress. Several pathogenic stress signals such as hypoxia, nutrient depletion, ER calcium ${ }^{21,22}$, and even viral infections can compromise ER function. Stressed cells trigger specific mechanisms to relieve ER stress and restore cellular homeostasis, one of which is the unfolded protein response (UPR).

The UPR aims to alleviate ER stress in three ways: (a) increasing the ER's protein-folding capacity by chaperone expression induction, (b) degrading unfolded proteins through ER-associated protein degradation pathways (ERAD), and (c) inhibiting general and specific ER protein translation. Finally, UPR can induce cell death when ER stress is irreparable ${ }^{23}$.

UPR induction is controlled by three major sensors emanating from the ER: (1) inositol-requiring protein 1 (IRE1), (2) PKR-like ER kinase, and (3) activating transcription factor $6^{24}$. All three are integral ER membrane proteins regulated by the main and most represented ER-resident chaperone BIP/GRP78, a $78 \mathrm{kDa}$ glucose-regulated protein. BIP/GRP78 usually prevents dimerization and activation by directly binding to their luminal domains ${ }^{25,26}$. When ER stress is detected, BIP/ GRP78 is released, leading to receptor dimerization and activation of the downstream signaling cascades activated by the three sensors (Fig. 2) 21,27,28. The UPR and ERAD pathways are fundamental processes for cell homeostasis; however, some viruses are capable of taking advantage of these functions to benefit their own life cycles.

\section{Hijacking the UPR and ERAD pathways during NV infection}

Replication cycles of RNA viruses are closely associated with the ER and cause ER membrane exploitation $^{29}$, accumulation of misfolded proteins, impair the balance of reactive oxygen species (ROS), alter calcium concentration, and sabotage or deplete the ER membrane during virion release ${ }^{21,30,31}$. Due to it, UPR is often skewed during virus infection and some RNA viruses are capable of disturbing the ER environment, controlling its function, and using it to benefit their replication cycles. Here, we present evidence of the potential role of NV in the UPR and ERAD pathways.

\section{NV and UPR modulation: ER exploitation}

The precise role NV play in UPR modulation during infection is as yet unknown. Recently, a study with MNV demonstrated that the interaction of ubiquitin-specific protease 14 and IRE1 is beneficial for MNV. Results obtained from treatment with deubiquitinase (DUB) 


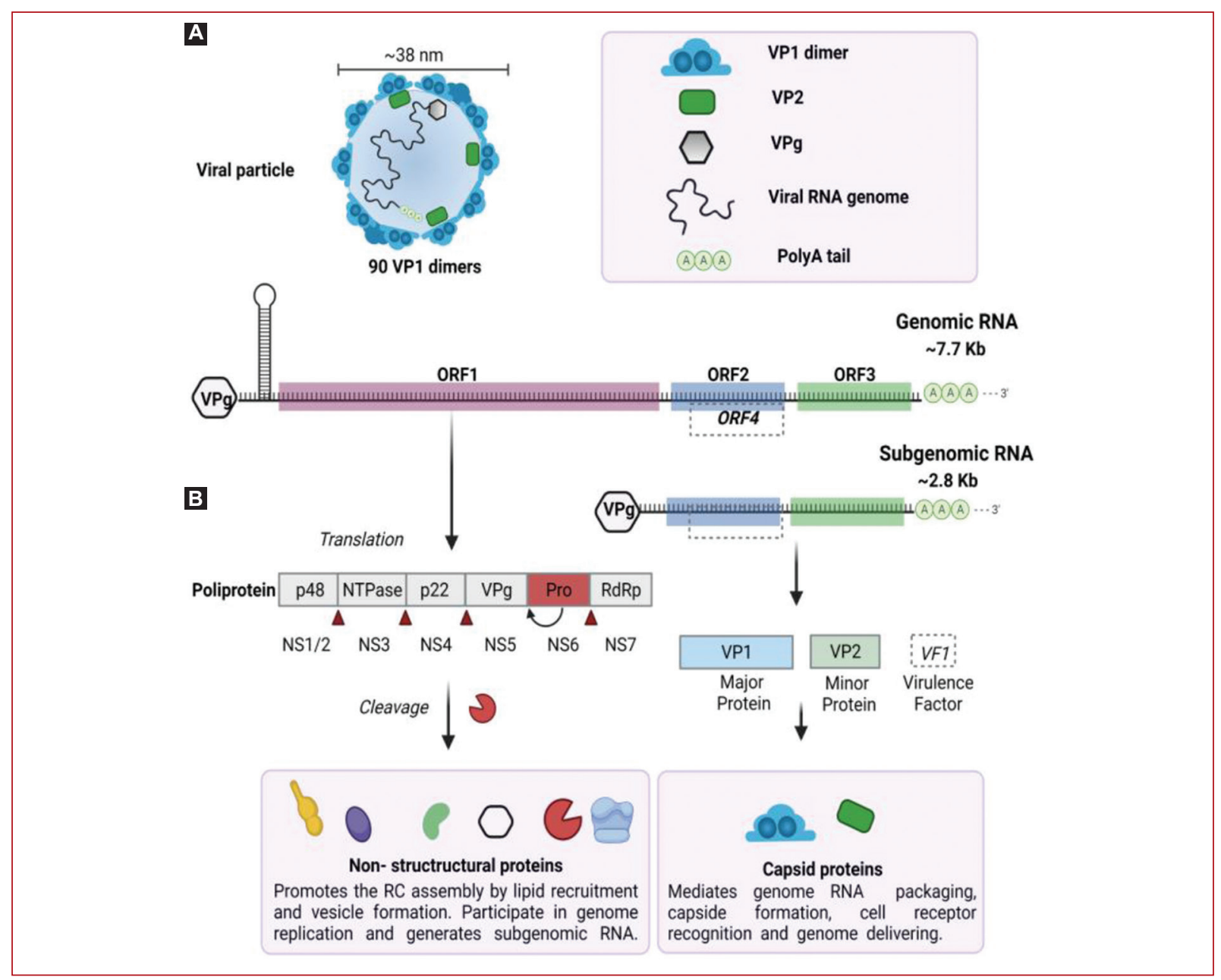

Figure 1. Molecular organization of human norovirus (HuNV). A: a schematic representation of the human norovirus virion. B: general scheme of the HuNV genome organization. The genome is an RNA (+) strand that encodes 3 ORFs. The ORF1 encodes the non-structural proteins: p48, NTPase, p22, VPg, Protease, and RdRp and can be translated directly by the ribosome after viral infection. Non-structural proteins form the replication complex, a membraneassociated structure where the genomic RNA is replicated, and subgenomic RNA is synthesized. Subgenomic RNA contains the ORF2 and ORF3 that encode the major structural protein (VP1) and the minor structural protein (VP2), respectively. In contrast to HuNV, Murine Norovirus contains an extra ORF (ORF4) that encodes for the virulence Factor (VF1).

inhibitor WP1130 compared with MNV alone significantly decreased MNV replication. In addition, treatment with irestatine, a specific inhibitor for IRE1, restored viral relocation. This effect was dependent on XBP1 splicing; however, it was unrelated to proteasome activity. In addition, treatment with UPR inductor thapsigargin showed wide spectrum antiviral properties, since similar results were also observed in other RNA viruses, such as the La Crosse virus, encephalomyocarditis virus, and Sindbis virus. Authors suggest that MNV-1 could prevent UPR activation in the early stages of the infection to gain membrane recruitment to its replication sites. Nonetheless, induction of the UPR can lead to inhibition of viral infection for viruses such as HCV or rotavirus $^{27,32}$. In addition, Passalacqua et al., in 2016, reported that C6, a compound derived from WP1130's molecular structure, also inhibits DUB activity. Experiments demonstrated the antiviral effect of C6 in MNV-1 infection within 4 and $8 \mathrm{~h}$ of infection. Since the ubiquitin system is involved in numerous cellular pathways possibly affected during viral infection, DUB's inhibition and its antiviral activity could be related to the restoration of cellular processes such as UPR activation or induction of the nitric oxide synthase in macrophages; whether there is a direct interaction of the UPR sensors, DUBs or viral proteins are still unknown $n^{33}$. 


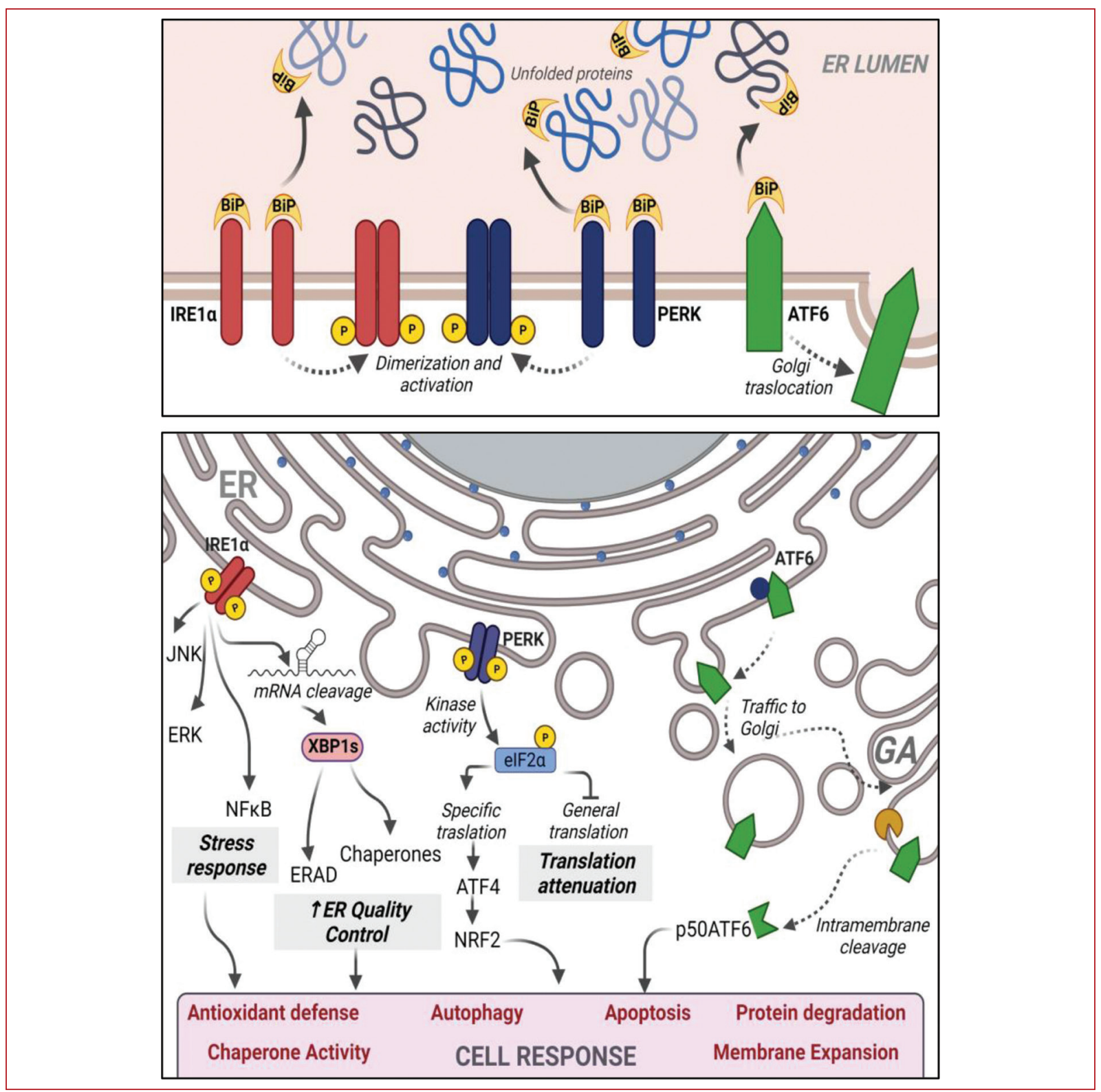

Figure 2. Unfolded protein response/endoplasmic reticulum (UPR/ER) stress signaling activation. IRE1 (red), PKR-like ER kinase (blue), and activating transcription factor 6 (green) are ER transmembrane proteins capable of sensing the level of unfolded or misfolded proteins in the ER lumen. Activation of each path requires the displacement of molecular chaperones such as BiP from the luminal domain of the protein (upper panel). UPR activation elicits antioxidant defense, chaperon activity, autophagy, apoptosis, protein degradation, and membrane expansion (lower panel).

In addition, using a riboproteomics-based approach, Vashist et al. in 2015 identified heat shock protein 90 (Hsp90) as one component of a network of host proteins that interact directly or indirectly with the $5^{\prime}$ and $3^{\prime}$ extremities of the MNV genome. Hsp90 is a chaperone required to properly fold diverse proteins, previously described to interact with IRE1, promoting its stability and playing an important role in UPR activation during
ER stress ${ }^{34}$. Experiments demonstrated that the major capsid protein VP1, of both MNV and HuNV, interacts with Hsp90. They demonstrated decreased VP1 levels and confirmed it as a client protein of Hsp90, and its inhibition was not related to VP1 degradation through proteasome or autophagy pathways; results were consistent with the analysis in vivo ${ }^{35}$. Inhibition of Hsp90 with a siRNA negatively impacts NV replication. Despite 
Hsp90 inhibition through its inhibitor geldanamycin (17DMAG) not affecting viral replication, a significant decrease in infectivity was reported.

Furthermore, another research group evaluated the effect of muramyl dipeptide, which is an activator of nucleotide-binding oligomerization domain-containing protein 2 that stimulates an innate response during MNV infection of bone marrow-derived macrophages. Results showed the activation of the MAPK pathways, particularly JNK, ERK, and p38, and NFk-B, which induced pro-inflammatory cytokines syntheses such as TNF- $\alpha$ and IL-6. Although these experiments did not focus on viral replication nor evaluated UPR sensors, MAPK pathway activation induced by MNV infection was demonstrated. Since IRE1 downregulates the MAPK pathway, the evaluation of IRE- 1 in this approach might bring a broader molecular scenario during NV infection ${ }^{36}$.

From these findings, we can observe (1) possible participation of NV in UPR modulation, particularly through the IRE1 arm and (2) a proven inhibition of NV under treatment with thapsigargin, which is a UPR activator, showing a potential interaction of NV with the UPR.

\section{ERAD modulation and major histocompatibility complex I (MHC-I) impairment: clues about immune evasion}

Today, there is little research related to the interaction of NV with the ERAD pathway; the information found today is presented here. First, a study demonstrated the influence of UPR over MHC-I. Since the UPR, besides protein-folding regulation, also regulates two key processes that control MHC-I: protein synthesis and degradation, therefore ${ }^{37}$, this demonstrates that UPR impinges MHC-I. Researchers performed experiments using the MHC-1 high-producing cells EL4 transfected to express a chimeric protein based on SIINFEKL peptide; they induced ER stress with palmitate or glucose starvation, probing increased mRNA levels of BIP, XBP1 , and $\mathrm{CHOP}$, demonstrating that ER stress induced by palmitate or glucose deprivation attenuates peptide presentation by MHC-I molecules and that these molecules are probably sequestered in the ER. Their hypothesis is, therefore, that global attenuation of protein synthesis caused by palmitate and glucose starvation limits the amount of a vast repertoire of peptides available for insertion in MHC-I molecules ${ }^{37}$.

In addition, Tuipulotu et al., in 2017, found consistent results regarding proteasome genes. After an NGS analysis evaluating the transcriptomic profile of MNV infected Raw264.7 cells in diverse stages of the infection, they found a significant perturbation of IFN signaling, viral recognition, cytokine stimulation, protein degradation, antigen presentation, and lymphocyte activation pathways ${ }^{38}$. Specifically, they found 69 differentially expressed genes downregulated solely by MNV infection, revealing an important impact on MHC-I molecular maturation, including proteolysis and vesicular trafficking. These genes, psmb3, psmb4, psmb5, and psmb9, which encode beta catalytic subunits of the $26 \mathrm{~S}$ proteasome, are important for viral protein degradation to produce antigenic peptides. Furthermore, psmb2 encodes the proteasome activator 28 subunit, which is an inducible protein that is involved in antigen processing by the immunoproteasome (i-proteasome), whose main function is to generate peptides with a hydrophobic $\mathrm{C}$ terminus that can be processed to fit in the groove of MHC-I molecules ${ }^{39}$. Furthermore, they found a decrease of Ap1s1, which encodes a subunit of adaptor protein 1, responsible for vesicular transport between the ER and Golgi, and a 24-fold subexpression of H2-Q10 which encodes Qa10, a secreted MHC-lb protein involved in immune cell activation ${ }^{38}$. Results obtained depict viral sabotage of immune recognition by preventing antigen processing in the i-proteasome.

These results corroborate a previous work published by the same research group, where they found MNV decreased MHC-I levels in MNV-infected Raw264.7 cells on a protein level since MHC-I mRNA level was not detected. They also found that proteasome inhibition by MG132 partially restores MHC-I detection, while virus replication maintains its levels with or without MG132. In addition, they found that NS3 was the MNV protein responsible for this effect in MHC-I proteasome-mediated degradation. In addition, they found that the MHC-I protein was associated with the inability of $C D 8+T$ cells to recognize 343 surface-expressed viral peptides and thus the infected cell ${ }^{40}$. This research group hypothesizes that MNV may be affecting protein trafficking and proteasome functions to limit the cellular capacity to communicate and respond to the infection itself. Finally, we can conclude that researchers have encountered consistent results regarding MNV influence in proteasomal subunits genes and its relevance in attenuating MHC-I function.

From these findings, we can observe (1) that innate response activation induces MAPK and pro-inflammatory cytokines during viral infection and (2) that the ER stress attenuates MHC-I (Fig. 2). 


\section{Conclusion}

NV is a health problem, with several ongoing studies to establish their pathogenesis. Nowadays, the potential influence of NV in ER control and the downregulation of innate immunity and MHC-1 are clearer. However, there are yet important points to investigate: how the NV induces ER stress and the precise pathways of UPR, proteasomal, or immune modulation. A clearer understanding of this will help improve therapies and designs and provide the tools to understand the molecular physiopathology of this viral disease.

\section{Funding}

This study was funded by PAICYT (SA1434-20).

\section{Conflicts of interest}

The authors declare that the research was conducted without any commercial or financial relationships that could be construed as a potential conflict of interest.

\section{Ethical disclosures}

Protection of human and animal subjects. The authors declare that no experiments were performed on humans or animals for this study.

Confidentiality of data. The authors declare that no patient data appears in this article.

Right to privacy and informed consent. The authors declare that no patient data appears in this article.

\section{References}

1. Pires SM, Fischer-Walker CL, Lanata CF, Devleesschauwer B, Hall AJ Kirk MD, et al. Aetiology-specific estimates of the global and regional incidence and mortality of diarrhoeal diseases commonly transmitted through food. PLoS One. 2015;10:1-17.

2. Koo HL, Ajami N, Atmar RL, Dupont HL. Noroviruses: the Principal Cause of Foodborne Disease Worldwide. Discov Med. 2010;10:61-70.

3. Ettayebi K, Crawford SE, Murakami K, Broughman JR, Karandikar U, Tenge VE, et al. Replication of human noroviruses in stem cell-derived human enteroids. Science. 2016;353:1387-93.

4. Robilotti E, Deresinski S, Pinsky BA. Norovirus. Clin Microbiol Rev. 2015;28:134-64

5. Glass RI, Parashar UD, Estes MK. Norovirus gastroenteritis. N Engl J Med. 2009;361:1776-85.

6. Chaudhry Y, Skinner MA, Goodfellow IG. Recovery of genetically defined murine norovirus in tissue culture by using a fowlpox virus expressing T7 RNA polymerase. J Gen Virol. 2007;88:2091-100.

7. Jones MK, Watanabe M, Zhu S, Graves CL, Keyes LR, Grau KR, et al. Enteric bacteria promote human and mouse norovirus infection of $B$ cells. Science. 2014;346:755-9.

8. Wobus CE, Karst SM, Thackray LB, Chang KO, Sosnovtsev SV, Belliot G, et al. Replication of norovirus in cell culture reveals a tropism for dendritic cells and macrophages. PLoS Biol. 2004;2:e432.

9. Desselberger U. Caliciviridae other than noroviruses. Viruses. 2019;11:286

10. Thorne LG, Goodfellow IG. Norovirus gene expression and replication. J Gen Virol. 2014:95:278-91.

11. Tu ET, Bull RA, Greening GE, Hewitt J, Lyon MJ, Marshall JA, et al. Epidemics of gastroenteritis during 2006 were associated with the spread of norovirus Gll.4 variants 2006a and 2006b. Clin Infect Dis. 2008:46:413-20.
12. Taube S, Perry JW, Yetming K, Patel SP, Auble H, Shu L, et al. Ganglioside-linked terminal sialic acid moieties on murine macrophages function as attachment receptors for murine noroviruses. J Virol. 2009;83:4092-101.

13. Nordgren J, Svensson L. Genetic susceptibility to human norovirus infection: an update. Viruses. 2019;11:226

14. Graziano VR, Wei J, Wilen CB. Norovirus attachment and entry. Viruses. 2019;11:495.

15. Orchard RC, Wilen CB, Doench JG, Baldridge MT, McCune BT, Lee YC, et al. Discovery of a proteinaceous cellular receptor for a norovirus. Science. 2016;353:933-6.

16. Haga K, Fujimoto A, Takai-Todaka R, Miki M, Doan YH, Murakami K, et al. Functional receptor molecules CD300lf and CD300ld within the CD300 family enable murine noroviruses to infect cells. Proc Natl Acad Sci U S A. 2016;113:E6248-55

17. Makino A, Shimojima M, Miyazawa T, Kato K, Tohya Y, Akashi H. Junctional adhesion molecule 1 is a functional receptor for feline calicivirus. J Virol. 2006;80:4482-90.

18. Orchard RC, Wilen CB, Virgin HW. Sphingolipid biosynthesis induces a conformational change in the murine norovirus receptor and facilitates viral infection. Nat Microbiol. 2018;3:1109-14.

19. Conley MJ, McElwee M, Azmi L, Gabrielsen M, Byron O, Goodfellow IG et al. Calicivirus VP2 forms a portal-like assembly following receptor engagement. Nature. 2019;565:377-81.

20. Corazzari M, Gagliardi M, Fimia GM, Piacentini M. Endoplasmic reticulum stress, unfolded protein response, and cancer cell fate. Front Oncol. 2017:7:1-11

21. Jheng JR, Ho JY, Horng JT. ER stress, autophagy, and RNA viruses. Front Microbiol. 2014;5:1-13.

22. Xue M, Fu F, Ma Y, Zhang X, Li L, Feng L, et al. The PERK arm of the unfolded protein response negatively regulates transmissible gastroenteritis virus replication by suppressing protein translation and promoting Type I interferon production. J Virol. 2018;92:1-36.

23. Tsai YC, Weissman AM. The unfolded protein response, degradation from the endoplasmic reticulum, and cancer. Genes Cancer. 2010;1:764-78.

24. Gardner BM, Pincus D, Gotthardt K, Gallagher CM, Walter P. Endoplasmic reticulum stress sensing in the unfolded protein response. Cold Spring Harb Perspect Biol. 2013:5:a013169.

25. Hetz C, Chevet E, Harding HP. Targeting the unfolded protein response in disease. Nat Rev Drug Discov. 2013;12:703.

26. Osorio $\mathrm{F}$, Lambrecht $\mathrm{BN}$, Janssens $\mathrm{S}$. Antigen presentation unfolded: identifying convergence points between the UPR and antigen presentation pathways. Curr Opin Immunol. 2018;52:100-7.

27. Perry JW, Ahmed M, Chang KO, Donato NJ, Showalter HD, Wobus CE. Antiviral activity of a small molecule deubiquitinase inhibitor occurs via induction of the unfolded protein response. PLoS Pathog. 2012;8:43.

28. Leestemaker $\mathrm{Y}$, Ovaa $\mathrm{H}$. Tools to investigate the ubiquitin proteasome system. Drug Discov Today Technol. 2017;26:25-31.

29. Hyde JL, Sosnovtsev SV, Green KY, Wobus C, Virgin HW, Mackenzie JM Mouse norovirus replication is associated with virus-induced vesicle clusters originating from membranes derived from the secretory pathway. J Virol. 2009;83:9709-19.

30. Gillespie LK, Hoenen A, Morgan G, Mackenzie JM. The endoplasmic reticulum provides the membrane platform for biogenesis of the flavivirus replication complex. J Virol. 2010:84:10438-47.

31. Gullberg RC, Steel JJ, Moon SL, Soltani E, Geiss BJ. Oxidative stress influences positive strand RNA virus genome synthesis and capping. Virology. 2015;475:219-29.

32. Ludert JE, Alcalá AC, Liprandi F. Primer pair p289-p290, designed to detect both noroviruses and sapoviruses by reverse transcription-PCR, also detects rotaviruses by cross-reactivity. J Clin Virol. 2004;42:835-6.

33. Passalacqua KD, Charbonneau ME, Donato NJ, Showalter HD, Sun D, Wen B, et al. Anti-infective activity of 2-cyano-3-acrylamide inhibitors with improved drug-like properties against two intracellular pathogens. Antimicrob Agents Chemother. 2016:60:4183-96.

34. Marcu MG, Doyle M, Bertolotti A, Ron D, Hendershot L, Neckers L. Heat shock protein 90 modulates the unfolded protein response by stabilizing RE1alpha. Mol Cell Biol. 2002;22:8506-13.

35. Vashist S, Urena L, Gonzalez-Hernandez MB, Choi J, de Rougemont A, Rocha-Pereira J, et al. Molecular chaperone Hsp90 is a therapeutic target for noroviruses. J Virol. 2015;89:6352-63.

36. Kim YG, Park JH, Reimer $T$, et al. Viral infection augments Nod1/2 signaling to potentiate lethality associated with secondary bacterial infections. Cell Host Microbe. 2012;9:496-507.

37. Granados DP, Tanguay PL, Hardy MP, et al. ER stress affects processing of MHC class I-associated peptides. BMC Immunol. 2009:10:1-15.

38. Tuipulotu DE, Netzler NE, Lun JH, Mackenzie JM, White PA. RNA sequencing of murine norovirus-infected cells reveals transcriptional alteration of genes important to viral recognition and antigen presentation. Front Immunol. 2017:8:1-15.

39. Ferrington DA, Gregerson DS. Immunoproteasomes: structure, function, and antigen presentation. Prog Mol Biol TransI Sci Author. 2012;109:75-112.

40. Fritzlar S, Jegaskanda S, Aktepe TE, Prier JE, Holz LE, White PA, et al. Mouse norovirus infection reduces the surface expression of $\mathrm{MHC}$ class I proteins and inhibits CD8+ $\mathrm{T}$ cell recognition and activation. J Virol. 2018;93:e00286-18. 